\title{
ESTIMATION OF DISTRIBUTION OF MOVING HEAT SOURCE DURING MILLING OF AISIH13 BY INVERSE HEAT CONDUCTION METHOD
}

\author{
SOMAYEH D. FARAhani \\ Mechanical Engineering Department, Arak University of Technology, Arak, Iran \\ e-mail: sdavoodabadifarahani@gmail.com
}

\begin{abstract}
This study presents an inverse heat transfer method to estimate the time history of a local heat flow into the work-piece during milling of AISIH13 with considering a 3D thermal model. Temperatures are measured using thermocouples within the work-piece providing input data for the inverse solver. The conjugate gradient method is used as an inverse solver to predict the local time dependent heat flow distribution on the cutting surfaces as well as the temperature distribution in the work-piece. A moving point heat source and a moving plane heat source with different heat source velocity is considered to investigate their influence on the estimated heat flow. Results indicate a good agreement between the experimental and estimated data with an average root mean square error less than $0.2^{\circ} \mathrm{C}$. It can be observed that the heat flow distribution is a function of heat source geometry, cutting speed and feed rate, but the temperature distribution is a weak function of geometry of the moving heat source. Changes of temperature with depth are studied. This study suggests that the developed inverse model can be successfully applied for estimating the heat flow and thermal field in the work-piece during milling.
\end{abstract}

Keywords: milling, inverse method, moving plane heat source, moving point heat source, cutting temperature

\section{Introduction}

Heat generation in the cutting operation is important in machining processes as it governs the economy of machining. Large amount of heat generated in the cutting zone causes inaccuracies and induces thermal damages in the part being machined. These problems can be even more serious when machining difficult-to-cut materials, especially materials with low thermal conductivity like titanium alloys (Shokrani et al., 2012), where excessive heat is conducted into the work-piece and makes a problem in the production. Therefore, reducing heat generation on the cutting surface is of great importance to guarantee excellent machined surface quality and precision. In order to do so, it is necessary to know heat sources and magnitudes of these heat flows. Most of these studies are related to modeling of the thermal phenomenon in the primary and secondary heat zones, and very few studies are focused on the work-piece. Almost all of these analytical and numerical modeling is based on simplified model assumptions, which causes a decrease in accuracy. As an illustration, a plastic material model for the work-piece as well as sharp cutting edges are mostly assumed when modeling machining processes, thus the effect of tertiary heat zone will be ignored when calculating the heat flow input to the work-piece. However, this assumption for worn tools cannot be considered valid anymore, and the tertiary heat zone must be taken into account. So, the best/convenient way to find the heat flow input to the work-piece is to measure it experimentally. Due to limitations and difficulties of direct measurement at the interfaces, inverse methods are suitable to estimate temperature and heat flow at the tool/work-piece interface. The inverse heat conduction problem involves parameter 
estimation (Kowsary and Farahani, 2010; Farahani et al., 2011) using some temperatures measured from locations within or on the surface of the body, and inverse design (Farahani et al., 2016). IHCPs are highly sensitive to random noise, inherently based on measured temperature data. In order to eliminate this problem, regularization methods are used (Beck et al., 1985). Lazard and Corvisier (2005) used an inverse heat transfer approach based on a quadruple formulation to predict temperature and heat flow at the tool tip in turning operation. Huang et al. (2007) developed a 3D thermal inverse model with an adjoint equation to predict heat flowing into the cutting tool during drilling operation of titanium. Considering the heat input to the work-piece, Tai et al. (2012) used an inverse method to study the heat flow on the wall surface of a hole in the drilling process. In another work on grinding operation, Gostimirović et al. (2011) applied an inverse method to find the temperature field and heat flow density distribution in the work-piece/wheel interface. A numerical approach using finite differences in an implicit form was used to solve the inverse problem. Luchesi and Coelho (2012) estimated the heat source in face milling of AISI 4340 steel. The mechanics of machining is complicated, and it is very difficult to predict the intensity and distribution of heat sources in a machining operation. During the machining operation, the material properties vary with temperature. Therefore, the machining process and the thermal dynamics process are fully coupled. Since the beginning of this century, many efforts have been made in theoretical analysis and experiments to understand this phenomenon, but many problems remained.

In order to be able to accurately estimate the distribution of heat produced in the work-piece during the machining operation, this research (see also Carvalho et al., 2006) is carried out according to the following points:

- The heat produced on the work-piece surface during the machining operation is a function of rotation of the machine and the tool, movement of the work-piece and the cutting speed. By advancing the tool on the work-piece, the amount of heat varies with location. Therefore, it is a function of time and space. So far, in the studies that have been done in this regard, this issue has not been considered.

- In most studies performed using the moving heat source method, the tool is considered as a moving point heat source, whereas in reality, the tool must be considered as a moving plane heat source. So far, this has not been considered in the studies done in this field. Also, the effect of the difference between two geometries for the heat source in generated heat estimation has not been investigated.

In the present study, an experiment is designed to estimate the temporal and spatial distribution of the produced heat in the work-piece during milling of AISI H13 and the effect of geometry of the moving heat source on the estimation. It is clear that temperature contains much information about heat generation on the cutting surface in the milling process. In order to obtain this information, it is necessary to decode the measured temperature using the inverse method. This paper uses the idea that the generated heat is estimated using the measurement of temperature and the inverse heat transfer method. The temperatures are measured within the work-piece using a thermocouple due to limitations and difficulties of direct measurement at the cutting surface. Thus, the $k$-type thermocouples inserted into specific locations within the work-piece are used to measure time dependent temperatures. The measured temperatures are used as input data in the inverse method to obtain heat generation in the work-piece. The conjugate gradient technique is employed to estimate the produced heat. A 3D transient thermal model in a half space of the work-piece is used. Motion of the tool on the work-piece is considered as a moving heat source. In order to evaluate the effect of heat source shape on the estimation, a point heat source and a plane heat source are considered. In fact, the novelty of the study is the inverse method developed to estimate the cutting temperature and time history of the local heat flow to the work-piece in milling of AISI H13 and investigation of the effect heat source geometry on the estimated heat flow. 


\section{Experimental apparatus}

Machining tests were carried out on a HERMLE C800U machining center with a maximum of $24000 \mathrm{rpm}$ using TiNAl coated carbide tool with $20 \mathrm{~mm}$ diameter and 8 teeth from Sandvik Coromant. The work-piece material was selected as hardened AISI H13 steel. A new tool was used for each milling condition to eliminate the influence of tool wear on the experiments consistency. All machining tests were fulfilled without application of a cutting fluid. The experimental setup used for temperature measurements is shown in Fig. 1a and illustrated schematically in Fig. 1b. $5 k$-type thermocouples with $1.25 \mathrm{~mm}$ wire diameter were used for the measurements. After the thermocouple arrangement was designed, holes with $1.5 \mathrm{~mm}$ diameter were created in specified positions to install the thermocouples. The thermocouples were embedded in the specific positions within the work-piece to measure the temperature.

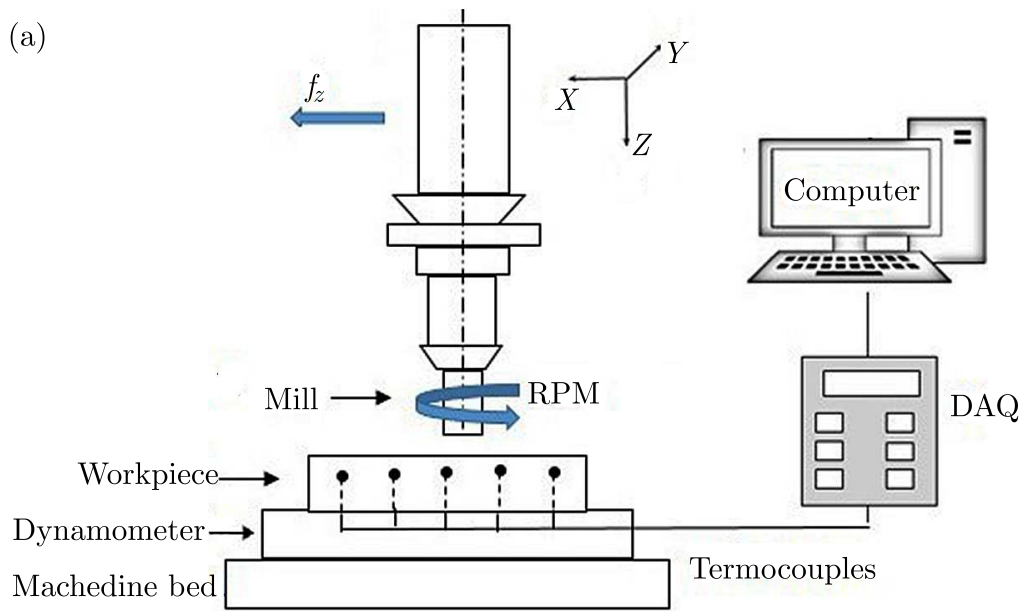

(b)
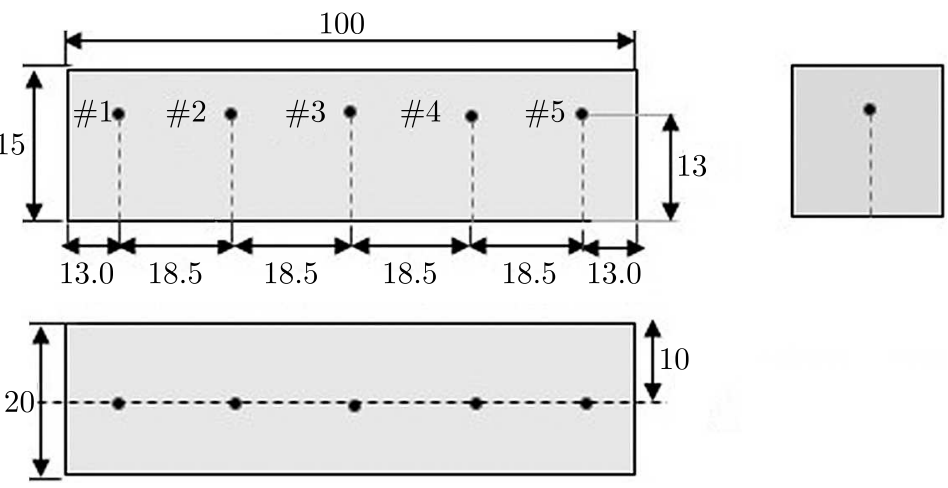

Fig. 1. (a) Experimental setup, and (b) thermocouple positions (dimensions in mm)

Table 1 lists location coordinates of the thermocouples according to the Cartesian coordinate system shown in Fig. 1b. The thermocouples were positioned $0.5 \mathrm{~mm}$ from the machined surface. The maximum spindle rotation, feed per tooth and axial depth of cut are $24000 \mathrm{rpm}, 0.02 \mathrm{~mm}$ and $0.1 \mathrm{~mm}$, respectively. A thermal compound of OMEGA "201" with a high thermal conductivity and low thermal resistance was used to minimize temperature losses. The temperature data were collected in every $0.1 \mathrm{~s}$ by a DT9805 data translation DAQ. The depth of cut was $0.1 \mathrm{~mm}$. Two cases were investigated. The first with the cutting speed and the feed rate of $50 \mathrm{~m} / \mathrm{min}$ and $100 \mathrm{~mm} / \mathrm{min}$, and the second with the cutting speed and the feed rate of $100 \mathrm{~m} / \mathrm{min}$ and $200 \mathrm{~mm} / \mathrm{min}$, respectively. 
Table 1. Thermocouple position coordinates

\begin{tabular}{|c|c|c|c|c|c|}
\hline Thermocouple No. & $T 1$ & $T 2$ & $T 3$ & $T 4$ & $T 5$ \\
\hline \hline$x[\mathrm{~mm}]$ & 13 & 31.5 & 50 & 68.5 & 87 \\
\hline$y[\mathrm{~mm}]$ & 0 & 0 & 0 & 0 & 0 \\
\hline$z[\mathrm{~mm}]$ & 0.5 & 0.5 & 0.5 & 0.5 & 0.5 \\
\hline
\end{tabular}

\section{Mathematical modeling}

Figure 2 shows the thermal model for the milling process, where the moving heat source method is capable of revealing thermal conductivity in orthogonal cutting. Two problems should be considered in thermal modeling:

- The semi-infinite solid provides a useful idealization for many practical problems. It may be used to approximate the transient response of a finite solid, such as a thick slab. In this second situation, the approximation would be reasonable for the early portion of the transient during which temperatures in the slab interior (a well removed from the surface) are essentially uninfluenced by the change in surface conditions (Jeager, 1942). Thus, according to this matter, thermal behavior of the work-piece in milling can be considered as the semi-infinite body. It is possible to refer to (Jeager, 1942) for further details.

- In this study, heat transfer Fourier's law is used. The effects of non-Fourier conduction are significant when the thermal relaxation time $\tau_{m}$ is large and this parameter depends on the material. The thermal relaxation time $\tau_{m}$ which must be very small (of the order of picoseconds for most metals), and the effects of non-Fourier conduction in this problem are negligible.

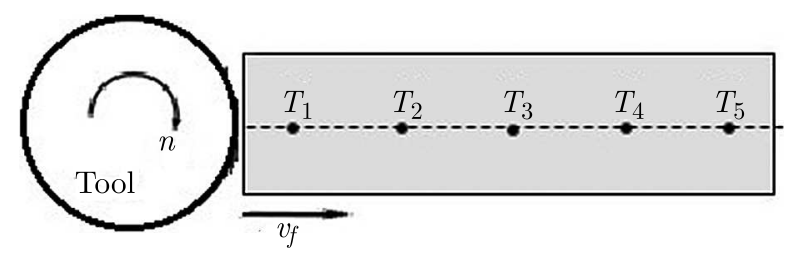

Fig. 2. Thermal model adopted for the milling process

As it happens at macro milling, the heat source is generated by formation of a chip along with friction of the tool on the material during milling. Two geometries for the moving heat source are considered: A moving point source and a moving plane source. In general, the heat equation in the Cartesian coordinate has the following form

$$
\frac{\partial^{2} \theta}{\partial x^{2}}+\frac{\partial^{2} \theta}{\partial y^{2}}+\frac{\partial^{2} \theta}{\partial z^{2}}=\frac{\rho C_{p}}{k} \frac{\partial \theta}{\partial t}
$$

where $\theta=T-T_{0}$ is the temperature rise, $T_{0}$ is the initial temperature, $k, \rho$ and $C_{p}$ are the thermal conductivity, density and heat capacity of the half space, respectively. Specific heat capacity, thermal conductivity and density are $460 \mathrm{~J} /(\mathrm{kg} \mathrm{K}), 28.6 \mathrm{~W} /(\mathrm{m} \mathrm{K})$ and $7760 \mathrm{~kg} / \mathrm{m}^{3}$, respectively. $\theta=0$, when $\sqrt{x^{2}+y^{2}+z^{2}} \rightarrow \infty$, and the prescribed amount of released heat is $q[\mathrm{~W}]$ through the point source. In fact, the heat transfer mechanism between the work piece and the environment is free convection. The value of the free convection heat transfer coefficient is approximately 10, with respect to variation of temperature, the amount of heat transfer is negligible $-k d \theta / d z=h\left(\theta-\theta_{\infty}\right) \cong 0$. All points except the point source are assumed to be adiabatic; i.e. $-k d \theta / d z=0$. 


\subsection{Moving point source}

If the point source shown in Fig. 3a moves with a constant velocity of $v$ in the $x$-direction, the temperature rise distribution (Jeager, 1942; Akbari et al., 2009) at the time $t$ due to the amount of heat released when the source was at $\left(v t^{\prime}, \gamma, 0\right)$ is

$$
d \theta=\frac{\dot{Q}}{4 \rho C_{p} \sqrt{(\pi \alpha)^{3}}} \frac{1}{\sqrt{\left(t-t^{\prime}\right)^{3}}} \exp \left[-\frac{\left(x-v t^{\prime}\right)^{2}+(y-\gamma)^{2}+z^{2}}{4 \alpha\left(t-t^{\prime}\right)}\right] d t^{\prime}
$$

where $Q[\mathrm{~W}]$ is the heat flow rate, $\alpha$ is the thermal diffusivity of the half space. The method to extract Eq. (3.2) in (Akbari et al., 2009) has been fully explained, which can be referred to for further details on that reference. Letting $\tau=t-t^{\prime}$, and for a constant heat flow rate and the properties, Eq. (3.2) yields

$$
\theta=\frac{\dot{Q}}{4 \rho C_{p} \sqrt{(\pi \alpha)^{3}}} \int_{0}^{t} \frac{1}{\sqrt{\tau^{3}}} \exp \left[-\frac{(x-v(t-\tau))^{2}+(y-\gamma)^{2}+z^{2}}{4 \alpha \tau}\right] d \tau
$$

Denoting $X=x-v t$ and $R=\sqrt{X^{2}+(y-\gamma)^{2}+z^{2}}$, which is the moving coordinate attached to the heat source at time, Eq. (3.3) reduces to

$$
\theta=\frac{\dot{Q} \exp \left(-\frac{v X}{2 \alpha}\right)}{4 \rho C_{p} \sqrt{(\pi \alpha)^{3}}} \int_{0}^{t} \frac{1}{\sqrt{\tau^{3}}} \exp \left(-\frac{R^{2}+v^{2} \tau^{2}}{4 \alpha \tau}\right) d \tau
$$

By changing variables, $\eta=v^{2} \tau /(4 \alpha)$, then Eq. (3.4) reduces to

$$
\theta=\frac{\exp \left(-\mathrm{Pe} X^{*}\right) \mathrm{Pe}}{4 \sqrt{\pi^{3}}} \int_{0}^{\beta} \frac{1}{\sqrt{\eta^{3}}} \exp \left[-\left(\frac{\varepsilon^{2}}{\eta}+\eta\right)\right] d \eta=\frac{\exp \left(-\mathrm{Pe} X^{*}\right) \mathrm{Pe}}{4 \sqrt{\pi^{3}}} I_{t}
$$

where $X^{*}=X / l$.

The integral in Eq. (3.5) has a closed form, and the dimensionless form of this equation is

$$
\theta\left(x^{*}, y^{*}, z^{*}, \mathrm{Fo}, \mathrm{Pe}\right)=\frac{\dot{Q}}{k l} \frac{\exp \left(-\mathrm{Pe} X^{*}\right) \mathrm{Pe}}{\sqrt{(4 \pi)^{3}}} I_{t} \quad I_{t}=\frac{\sqrt{\pi}}{\mathrm{Pe} R^{*}}\left(I_{1}+I_{2}\right)
$$

where

$$
\begin{aligned}
& x^{*}=\frac{x}{l} \quad y^{*}=\frac{y}{l} \quad z^{*}=\frac{z}{l} \quad R^{*}=\frac{R}{l} \\
& \mathrm{Fo}=\frac{\alpha t}{l^{2}} \quad \mathrm{Pe}=\frac{v l}{2 \alpha} \\
& I_{1}=\exp \left(\mathrm{Pe} R^{*}\right) X^{*}=X / l \operatorname{erfc}\left(\frac{R^{*}}{2 \sqrt{\mathrm{Fo}}}+\mathrm{Pe} \sqrt{\mathrm{Fo}}\right) \\
& I_{2}=\exp \left(-\mathrm{Pe} R^{*}\right) X^{*}=X / l \operatorname{erfc}\left(\frac{R^{*}}{2 \sqrt{\mathrm{Fo}}}-\mathrm{Pe} \sqrt{\mathrm{Fo}}\right)
\end{aligned}
$$

The Péclet number Pe appears in Eq. (3.6) $)_{1}$ is the ratio of heat source velocity to the amount of heat diffuses into the half space, and the Fourier number Fo is the dimensionless time. 


\subsection{Moving plane heat source}

Consider a plane moving source having an ellipse shape as shown in Fig. 3b. An elliptical heat source is represented by

$$
\left(\frac{x}{a}\right)^{2}+\left(\frac{y}{b}\right)^{2}=1
$$

where $a$ and $b$ are the major and minor axis, respectively. The solution of the moving point source (Akbari et al., 2009) can be extended to the plane heat source by superimposing the temperature rise due to each moving infinitesimal element. Hence

$$
\theta\left(x^{*}, y^{*}, z^{*}, \mathrm{Fo}, \mathrm{Pe}\right)=\frac{\dot{Q}}{k l} \frac{\mathrm{Pe}}{\sqrt{(4 \pi)^{3}}} \int_{-a^{*}}^{a^{*}} \int_{-B}^{B} \exp \left[-\operatorname{Pe}\left(x^{*}-\chi^{*}\right)\right] I_{t} d \gamma^{*} d \chi^{*}
$$

where

$$
B=\varepsilon a^{*} \sqrt{1-\left(\frac{\chi^{*}}{a^{*}}\right)^{2}} \quad \gamma^{*}=\frac{\gamma}{l} \quad \chi^{*}=\frac{\chi}{l} \quad a^{*}=\frac{a}{l}
$$

and $\varepsilon=b / a$ is the aspect ratio. In this study, $\varepsilon$ is 1 and $a$ is $10 \mathrm{~mm}$. This implies that a certain state of the ellipse $(a=b=10 \mathrm{~mm})$ is actually the circle. A computational code has been developed in MATLAB to solve Eq. (3.8) numerically. In direct thermal problems, all boundary conditions and material properties are known. However, the heat flow rate is unknown, and instead of the heat flow rate, measured temperatures by thermocouples are available from an experimental test. Table 1 shows the position of thermocouples within the work-piece. The inverse method, conjugated gradient method (CGM), estimates the local heat flow into the work-piece using the measured temperature data recorded from specified locations in the workpiece.

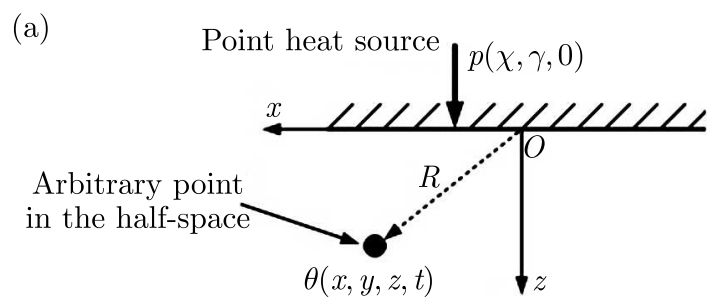

(b)

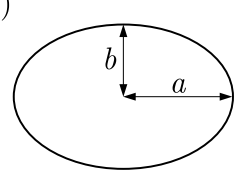

Fig. 3. (a) Schematic of an arbitrary shape moving plane source, and (b) an elliptical heat source

\section{Conjugated Gradient Method (CGM)}

One of the iterative approaches to solving IHCPs is the conjugate gradient method (Farahani et al., 2014). This method is very accurate and rapid. In this method, the goal is to minimize the error-squares function. The error-squares function $S$ is defined as

$$
S(q)=\sum_{i=1}^{N_{s}} \sum_{m=1}^{N_{m}}\left[Y_{i}\left(t_{m}\right)-T_{i}\left(t_{m}\right)\right]^{2}
$$

where $N_{s}$ and $N_{m}$ are, respectively, numbers of sensors and time. $Y$ and $T$ are, respectively, the measured and estimated temperatures at sensor locations in the heat equation. The heat flow into the work-piece is estimated by using the conjugate gradient method. To $T_{0}$ prevent 


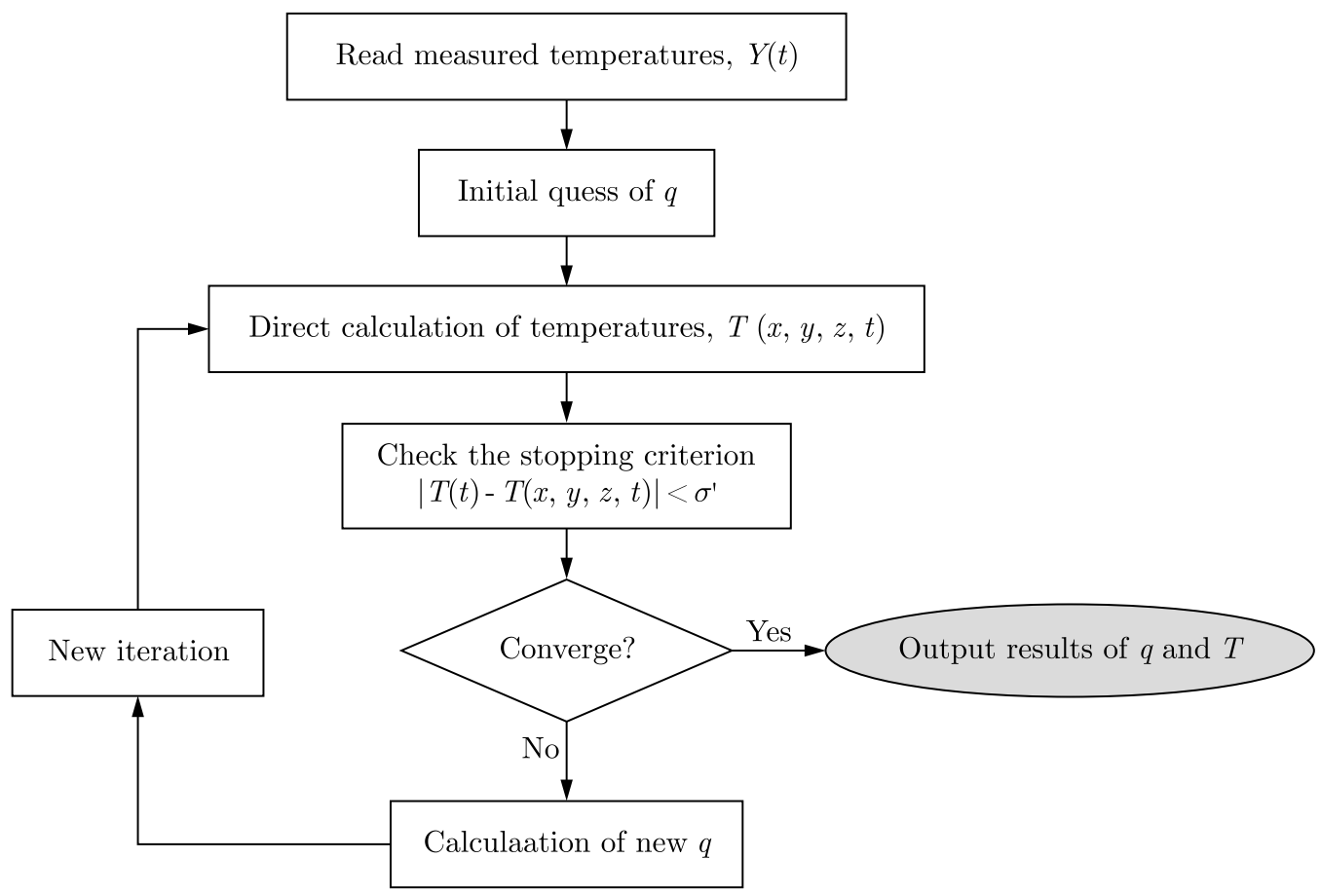

Fig. 4. Iterative procedure for IHCP

repetition, complete details and algorithms of this method are in (Farahani et al., 2014). The iterative procedure for the inverse method is shown in Fig. 4.

The spatial variation of the heat source is estimated by using the measured temperatures at measured points on the cutting surface. Five parameters: $q_{1}, q_{2}, \ldots, q_{5}$ are utilized to describe heat flow on the cutting surface; these parameters vary with time. Before using the proposed method, its accuracy should be determined. Error analysis is performed considering AISI H13 as the work-piece material, heat flow of $150 \mathrm{~W}$, initial work-piece temperature of $23^{\circ} \mathrm{C}$ and the heat source velocity of $100 \mathrm{~m} / \mathrm{min}$. It should be noted that the heat source velocity is the same as the cutting velocity. Direct temperatures calculated as a result of a known imposed heat flow are perturbed by a Gaussian noise with standard deviation $\sigma=0.1^{\circ} \mathrm{C}$. These temperatures were taken as experimental temperatures and used as the input data in the inverse algorithm to estimate the heat flow on each interval. The time step used in the direct and inverse calculations is $0.01 \mathrm{~s}$ and $0.1 \mathrm{~s}$, respectively. The root mean square error is used to compare estimated values with actual values of heat flow. This error is calculated as

$$
\mathrm{RMS}=\sqrt{\frac{1}{N} \sum_{i=1}^{N}\left(\widehat{q}_{i, \text { npisydata }}-q_{i}\right)^{2}}
$$

where $\widehat{q}_{i, \text { npisydata }}$ is the heat flow estimated by the inverse method. The mean value of the estimated heat flow is $147 \mathrm{~W}$ and $141 \mathrm{~W}$ for the moving point heat source and moving plane heat source, respectively. RMS in this error analysis is $0.01 q_{\text {mean }}$ and $0.02 q_{\text {mean }}$ for the point and plane heat source. Errors in the moving plane heat source are greater than errors in the moving point heat source. The variance error shows that the plane heat source is more sensible to measurement errors. In order to clarify this matter, sensivity analysis has been performed. The sensitivity coefficient for the heat flow is defined as

$$
X_{p}\left(x_{j}, Y_{j}, t_{m}\right)=\frac{\partial T\left(x_{j}, y_{j}, t_{m}\right)}{\partial q_{p}^{m}} \quad \begin{aligned}
& p=1,2, \ldots, P \\
& j=1,2, \ldots, J \\
& m=1,2, \ldots, n
\end{aligned}
$$


The sensitivity coefficient, Eq. (4.3), in the inverse method is the variation of temperature with respect to the variation unknown parameter. The sensitivity coefficient actually shows the difficulty of the inverse problem in the estimation of the unknown parameter. A smaller sensitivity coefficient causes greater difficulty and error in the estimation of the unknown parameter. Considerable insight can be found in the estimation problem by careful analysis of the sensitivity coefficient. Figures 5 and 6 show the sensitivity coefficient time-histories for $v=50 \mathrm{~m} / \mathrm{min}$ and $v=100 \mathrm{~m} / \mathrm{min}$, respectively. As can be seen, the sensitivity coefficient is a function of geometry of the heat source and its velocity. The value of the sensitivity coefficient for the point heat source is larger than for the plane heat source. The sensitivity coefficients are sensitive to the change in the heat source velocity, and their values decrease with increasing velocity. The estimation problem can be hard and sensitive to measurement errors if the value of the coefficients of sensitivity is small. This will reduce the accuracy of the problem. Comparison between the errors of two different heat sources in error analysis is provided, and it is observed that the moving point heat source gives better results and has less error. Therefore, the inverse problem for the moving plane source with greater velocity is more sensitive to measurement errors.

(a)

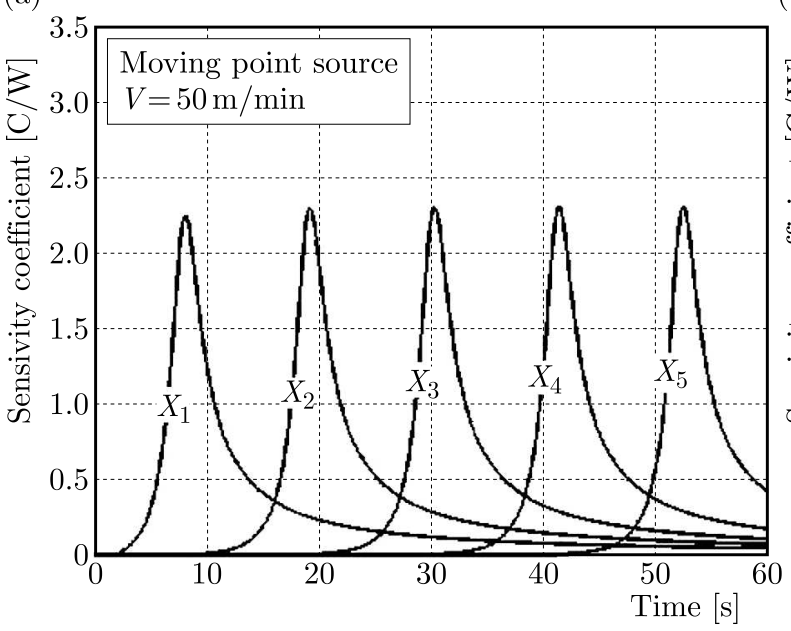

(b)

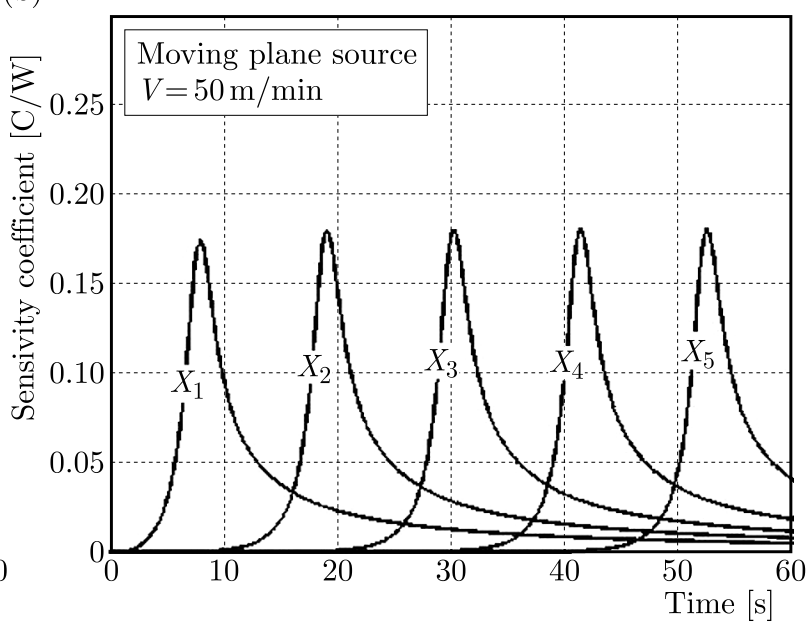

Fig. 5. Sensivity coefficient profiles at measuring points for $v=50 \mathrm{~m} / \mathrm{min}$ : (a) moving point heat source and (b) moving plane heat source

(a)

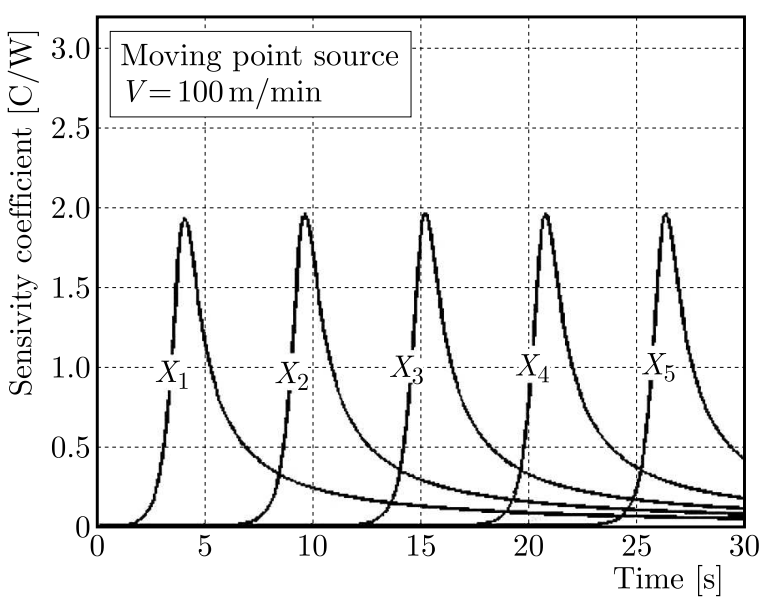

(b)

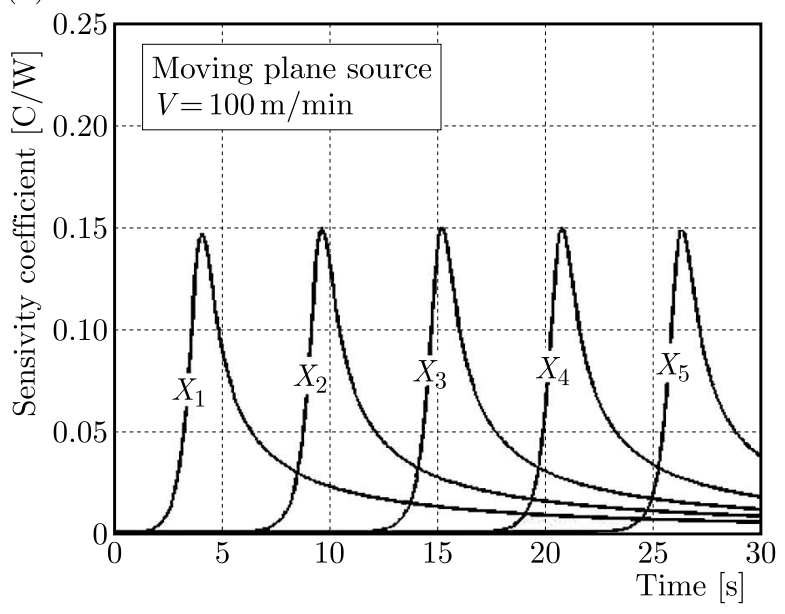

Fig. 6. Sensivity coefficient profiles at measuring points for $v=100 \mathrm{~m} / \mathrm{min}$ : (a) moving point heat source and (b) moving plane heat source 


\section{Results and discussion}

It should be noted in the inverse heat conduction problems that the accuracy of estimation of the unknown parameter depends on the distance of thermocouples from the active surface (the surface of heat produced on it) (Kowsary and Farahani, 2010; Farahani et al., 2011). If the thermocouples are closer to the active surface, the greater accuracy will be. In this study, according to the limitations, the thermocouples are in $0.5 \mathrm{~mm}$ from the active surface (or cutting surface). The transient temperature histories during the milling process at the determined points were measured for the cutting speed $v=50 \mathrm{~m} / \mathrm{min}$ and $100 \mathrm{~m} / \mathrm{min}$ (Fig. 7). The cutting time for

(a)

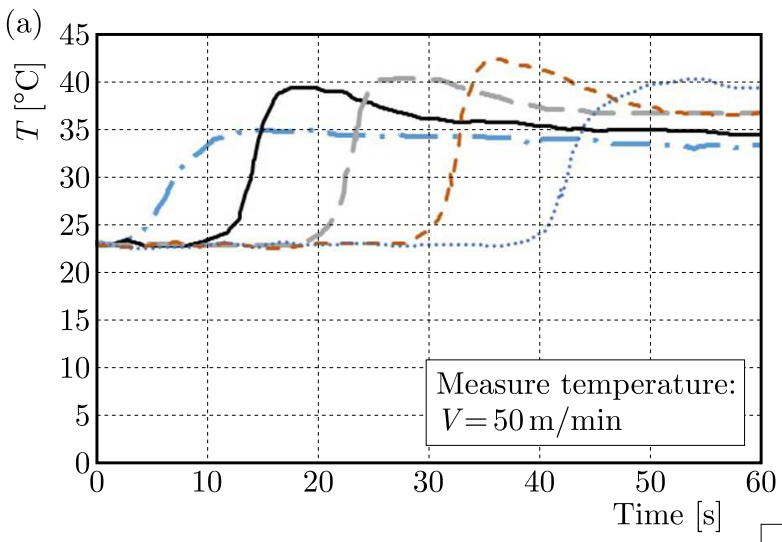

(b)

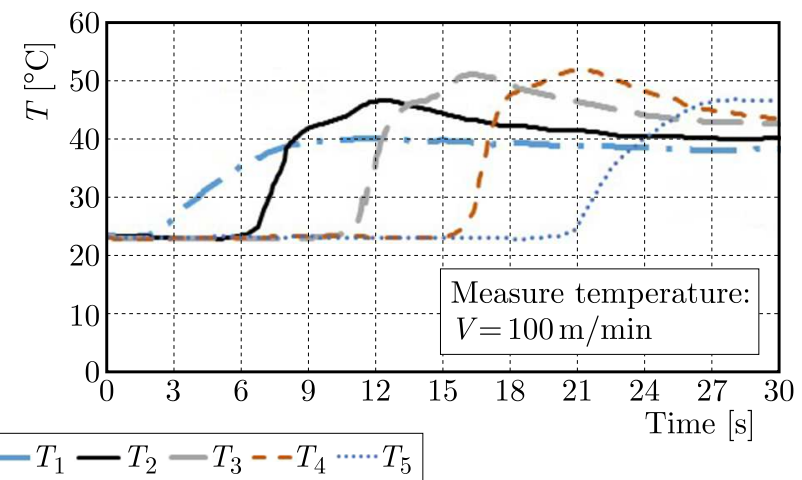

Fig. 7. Experimental temperatures in thermocouples: (a) $v=50 \mathrm{~m} / \mathrm{min}$, and (b) $v=100 \mathrm{~m} / \mathrm{min}$

$v=50 \mathrm{~m} / \mathrm{min}$ and $100 \mathrm{~m} / \mathrm{min}$ was $60 \mathrm{~s}$ and $30 \mathrm{~s}$, respectively. Using the cutting time, the feed rate can be calculated as feed rate $[\mathrm{mm} / \mathrm{min}]=$ Cut length $[\mathrm{mm}] /$ Cutting time $[\mathrm{min}]$. Temperature increases with the milling time due to passing of the tool over work-piece length. With the tool approaching to the measuring points, the machined surface warms up to a peak value $\left(T_{\max }\right)$ and then, after passing the thermocouples, cools down. Temperature decreases in 5 -th thermocouple in both experiments with different cutting speeds. 5-th thermocouple is placed close to the work-piece edge. There is no enough time to understand the variation of heat generation at that point and achievement of the maximum temperature. The temperature peak increases with the moving direction due to heat addition as the tool travels over work-piece length. The measured temperatures contain much information about the heat flow into the work-piece. In order to obtain this information, it is necessary to decode the measured temperatures using the reverse method. The measured temperatures are used as the input data in the inverse method to obtain the heat flow into the work-piece.

Figure 8 shows the results estimated by the inverse method with the experimental transient temperature profiles, presenting a good match between them. It is, therefore, concluded that the purposed inverse model is suitable for thermal modeling of the milling process in this work. The current deviation between the measured and estimated temperatures might arise due to assumptions made in numerical solution or uncertainty sources like the thermocouple position, measuring accuracy of the thermocouples or thermal properties of the work-piece, which may affect the results. It is important to know magnitudes of the heat flow in order to assess the machining process heat (Putz et al., 2015; Veiga et al., 2013). Optimal machining free of thermal defects in the work-piece requires methods for optimizing thermal phenomena by regulation of cutting temperature which itself is linked to the heat flow. Therefore, milling tests were performed to determine the generated heat flow. Based on the measured temperatures, the net heat flowing into the work-piece is estimated. The net heat flow is the difference between the heat generated by the cutting tool during the milling process and the heat transferred to the 
surrounding fluid. Convective heat coefficient is a function of the cutting speed, spindle rotation and the mill tool of the machine tool. The exact amount can not be determined, therefore, the inverse method estimates the net heat flow $q$.

(a)

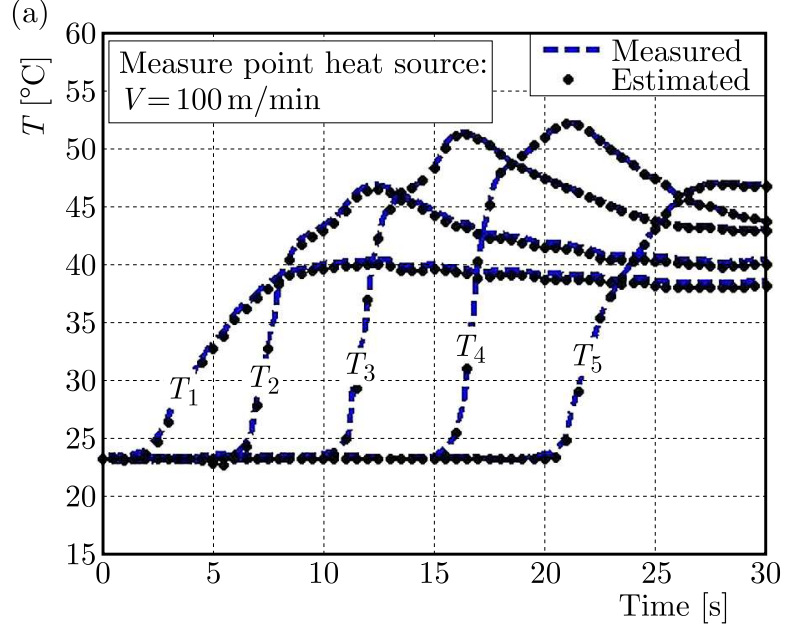

(b)

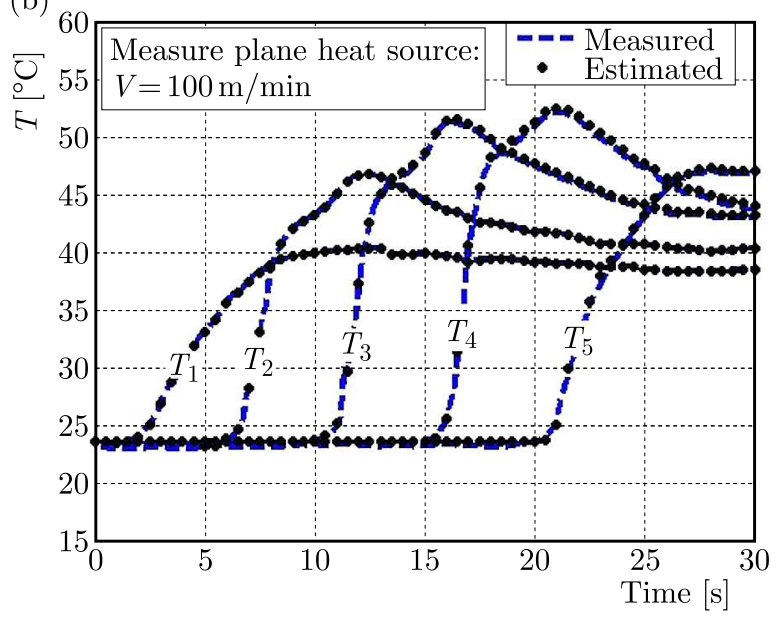

Fig. 8. Comparison of the estimated and measured temperature profiles at measuring points for $v=100 \mathrm{~m} / \mathrm{min}$ : (a) moving point heat source, and (b) moving plane heat source
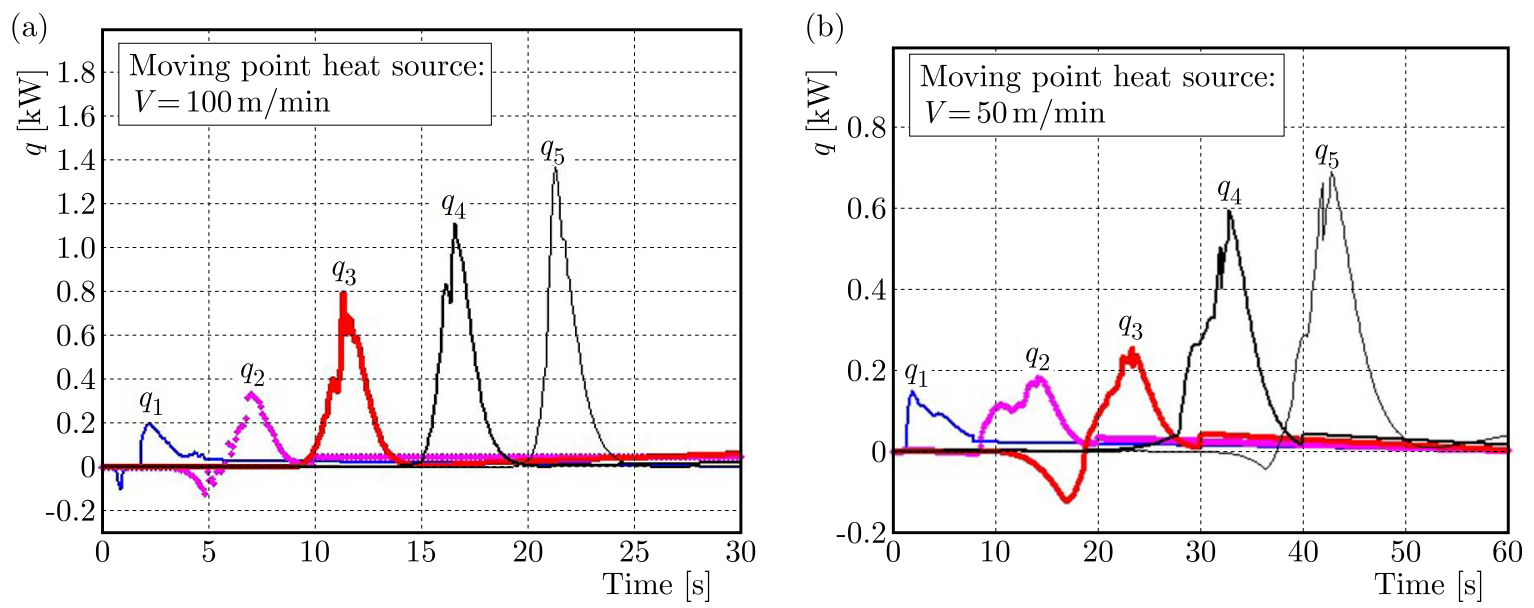

Fig. 9. Estimated heat flow distribution for the moving point heat source: (a) $v=100 \mathrm{~m} / \mathrm{min}$, and (b) $v=50 \mathrm{~m} / \mathrm{min}$

Figures 9 and 10 show the estimated heat flow for the moving point and moving plane heat source. The estimated heat flow is proportional to the measured temperature. The estimated net heat flow increases with the cutting tool approaching the measuring points to arrive of the maximum value. As the cutting tool moves away from the point of measurement, the estimated net heat flow decreases. The estimated net heat flow is less than zero when the amount of heat transferred to the surrounding fluid (air) is greater than the generated heat by the cutting tool. The effect of moving source geometry on the estimated heat flow is studied. The estimated heat flow for the moving plane heat source is nearly 10 times greater than for the moving point heat source. In the moving plane heat source, it is assumed that the cutting tool is similar to the moving elliptical heat source, and the generated heat during the milling process is transferred to the work-piece from the elliptical surface. The estimated maximum heat flow increases on the cutting surface in the moving heat source direction. The effect of the heat source speed on the temperature distribution of the work-piece is addressed in various applications. The effect 
(a)

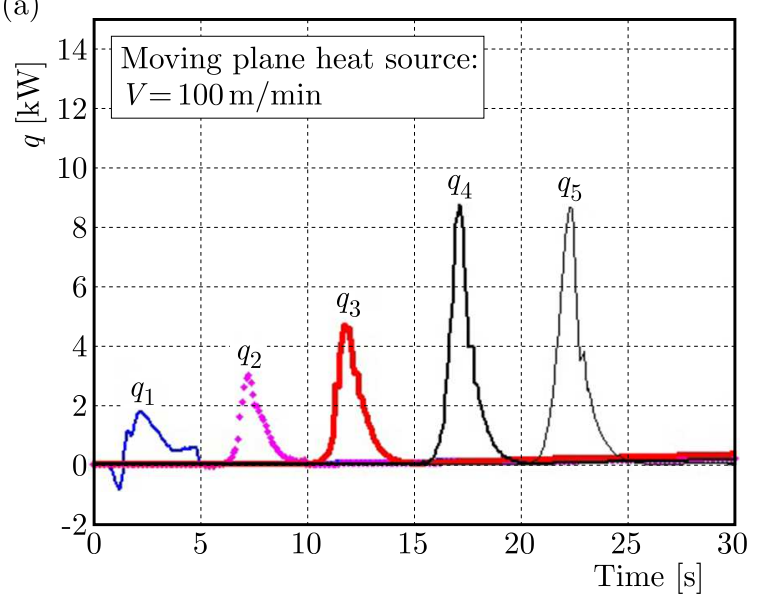

(b)

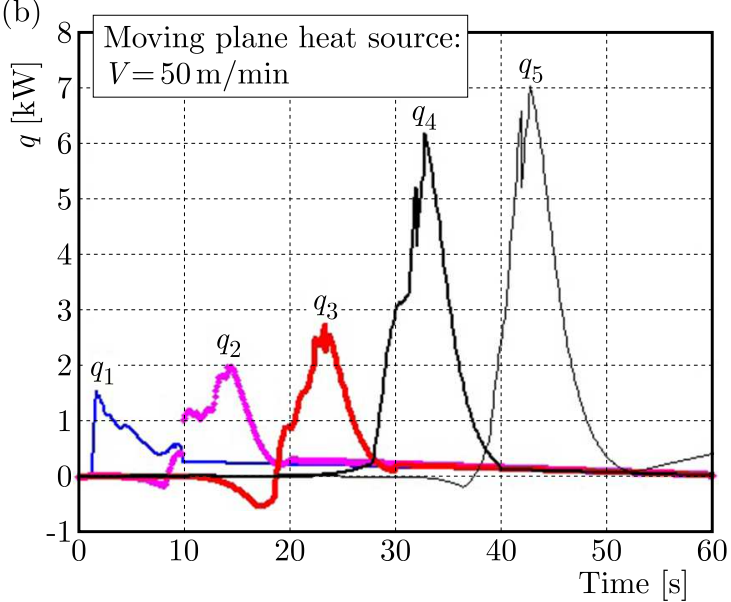

Fig. 10. Estimated heat flow distribution for the moving plane heat source: (a) $v=100 \mathrm{~m} / \mathrm{min}$, and (b) $v=50 \mathrm{~m} / \mathrm{min}$

of the heat source velocity on the estimations is investigated. The net heat flow increases with the increasing cutting speed and feed rate independent of the source shape modeling used. This behavior takes place given the greater heat input into the work-piece due to the high shear rate during chip formation and friction of the tool on the material. It should be recalled that at the cutting speed of $100 \mathrm{~m} / \mathrm{min}$, in addition to cutting speed, the feed rate doubles. In this state, the amount of increase in the heat flow is more than 1.5 times.

(a)

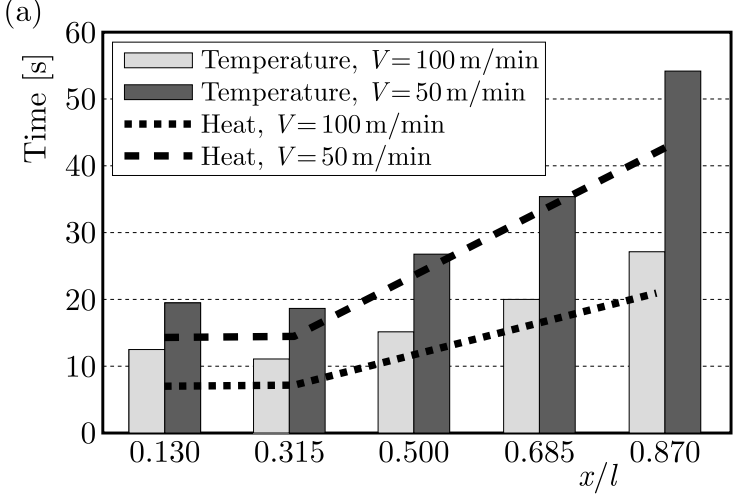

(b)

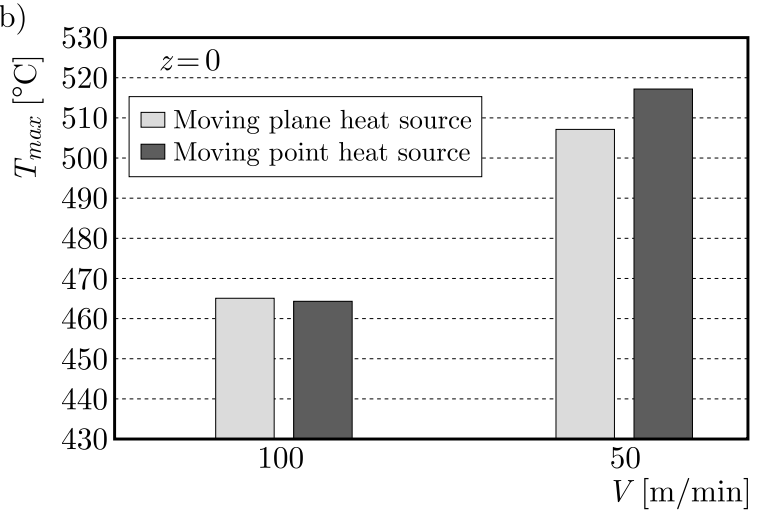

Fig. 11. (a) The occurred time for $T_{\max }$ and $q_{\max }$ in the moving direction and (b) variation of estimated maximum temperatures in the work-piece at the point $(50 \mathrm{~mm}, 0 \mathrm{~mm}, 0 \mathrm{~mm})$

Figure 11a shows the times in which the maximum heat flow and the maximum temperatures were observed. The results show that when the maximum heat flow is applied, the maximum temperature is not reached. One observes the time required for the work-piece to respond to the changes in the heat flow on the cutting surface and its temperature changes. The generated heat during cutting tests, estimated in the previous Section, were entered into the milling thermal model in order to estimate the work-piece temperature close to the cut. The variations of estimated maximum temperatures in the work-piece at the point $(50 \mathrm{~mm}, 0 \mathrm{~mm}, 0 \mathrm{~mm})$ is shown in Fig. 11b. As can be seen, the cutting temperature is independent of the heat source geometry. The cutting surface temperature is a function of the cutting velocity and feed rate. Its value for $v=50 \mathrm{~m} / \mathrm{min}$ and $v=100 \mathrm{~m} / \mathrm{min}$ is $510^{\circ} \mathrm{C}$ and $464.9^{\circ} \mathrm{C}$, respectively. At greater cutting speeds and feed rates, the time of heat transfer is shorter, and the cutting surface temperature for $v=100 \mathrm{~m} / \mathrm{min}$ is less than for $v=50 \mathrm{~m} / \mathrm{min}$. It can be observed that temperature drops rapidly at points near the heat source surface followed by a gradual change with increasing depth. This temperature 
gradient shows that the effect of heat source geometry on the maximum temperature is small and can be neglected.

\section{Conclusions}

Time and space distribution of the heat flow into the work-piece on the cutting surface in macromilling has been investigated. Assuming that motion of the tool on the work-piece is like a moving heat source, the work-piece is modeled with a semi-infinite body with a moving heat source. Using measured temperatures inside the work-piece and using an inverse heat conduction method, distribution of heat generation at the cutting surface is estimated. To investigate the effect of heat source geometry, a point heat source and a plane heat source are considered. The investigations are carried out for two states with different cutting speeds and feed rates. The conjugate gradient method (CGM) is used. The temperatures within the work-piece were experimentally measured providing the input data for the inverse method to estimate time the history of the local heat flow on the cutting surfaces. From the error analysis and calculation of the sensitivity coefficient, it was found that the moving point heat source had less error. RSM error between the estimated and measured temperatures is less than $0.2^{\circ} \mathrm{C}$. The estimated heat flow into the work-piece is a function of the heat source geometry, the cutting speed and feed rate. The estimated heat flow for the moving plane heat source is more than 10 times greater than the moving point heat source. Independent of the heat source shape, the maximum amount of heat generation increases with the increasing cutting speed and feed rate due to the high shear rate during chip formation. For both shapes of the heat source, the maximum heat generation value increases when the cutting speed and feed rate doubles. In all cases, the mount of the maximum heat increases in the direction of movement of the tool on the work-piece. Using the estimated heat generation, temperatures within the work-piece were also calculated. The calculated temperature on the cutting surface (the cutting temperature) is independent of the heat source geometry and depends on the cutting speed and feed rate. This temperature decreases when the cutting speed and feed rate doubles. The temperature distribution is a weak function of the shape of the heat source. Finally, the results obtained in this study suggest that the developed inverse model can successfully be used for predicting the thermal field in the work-piece, helping one to understand the possible phase transformations or thermal softening/hardening. The findings of this paper can be developed to other work materials, cooling systems and machining operations, i.e. turning and grinding.

\section{References}

1. Akbari M., Sinton D., Bahrami M., 2009, Moving heat sources in a half space: effect of source geometry, Proceedings of the ASME 2009 Heat Transfer Summer Conference HT2009

2. Beck J.V., Blackwell B., Clair C.S., 1985, Inverse Heat Conduction: Ill-Posed Problems, Wiley-Interscience, New York, NY, USA

3. Carvalho S.R., Lima e Silva S.M.M., Machado A.R., Guimaraes G., 2006, Temperature determination at the chip-tool interface using an inverse thermal model considering the tool and tool holder, Journal of Materials Processing Technology, 179, 1, 97-104

4. Farahani S.D., Bijarchi M.A., Kowsary F., Ashjaee M., 2016, Optimization arrangement of two pulsating impingement slot jets for achieving heat transfer coefficient uniformity, Journal of Heat Transfer, 138, 10, 102-110

5. Farahani S.D., Kowsary F., Jamili J., 2014, Direct estimation local convective boiling heat transfer coefficient in mini channel by using conjugated gradient method with adjoint equation, International Communication of Heat and Mass Transfer, 55, 1-7 
6. Farahani S.D., Sefidgar M., Kowsary F., 2011, Estimation of kinetic parameters of composite materials during the cure process by using wavelet transform and mollification method, International Communications in Heat and Mass Transfer, 38, 9, 1305-1311

7. Gostimirović M., Sekulić M., Kopač J., Kovač P., 2011, Optimal control of work-piece thermal state in creep-feed grinding using inverse heat conduction analysis, Strojniški Vestnik Journal of Mechanical Engineering, 57, 10, 730-738

8. Huang C.H., Jan L.C., Li R., Shin A.J., 2007, A three-dimensional inverse problem in estimating the applied heat flow of a titanium drilling-theoretical and experimental studies, International Journal of Heat and Mass Transfer, 50, 17, 3265-3277

9. JeAger J.C., 1942, Moving sources of heat and temperature at sliding contacts, Processding of Royal Society, New South Wales, 76, 203-224

10. Kowsary F., Farahani S.D., 2010, The smoothing of temperature data using the mollification method in heat flow estimating, Numerical Heat Transfer, Part A: Applications, 58, 3, 227-246

11. Lazard M., Corvisier P., 2005, Inverse method for transient temperature estimation during machining, Proceedings of the 5th International Conference on Inverse Problems in Engineering: Theory and Practice

12. Luchesi V.M., Coelho R.T., 2012, An inverse method to estimate the moving heat source in machining process, Applied Thermal Engineering, 45, 64-78

13. Putz M., Schmidt G., Semmler U., Dix M., Bräunig M., Brockmann M., Gierlings S., 2015, Heat flow in cutting: importance, simulation and validation, Procedia CIRP, 31, 334-339

14. Shokrani A., Dhokia V., Newman S.T., 2012, Environmentally conscious machining of difficult-to-machine materials with regard to cutting fluids, International Journal of Machine Tools and Manufacture, 57, 83-101

15. Tai B.L., Stephenson D.A., Shin A.J., 2012, An inverse heat transfer method for determining work-piece temperature in minimum quantity lubrication deep hole drilling, Journal of Manufacturing Science and Engineering, 134, 2, 206-210

16. Veiga C., Davim J.P., Loureiro A.J.R., 2013, Review on machinability of titanium alloys: the process perspective, Reviews on Advanced Materials Science, 34, 148-164 